\title{
A UK nationwide study of people with type 1 diabetes admitted to hospital with COVID-19 infection
}

\author{
Yue Ruan ${ }^{1,2} \cdot$ Robert E. J. Ryder $^{3} \cdot$ Parijat De $^{3} \cdot$ Benjamin C. T. Field ${ }^{4,5} \cdot$ Parth Narendran $^{6,7} \cdot$ Ahmed Iqbal $^{8}$. \\ Rajiv Gandhi ${ }^{8}$. Sophie Harris ${ }^{9}$. Dinesh Nagi ${ }^{10}$. Umaira Aziz ${ }^{11}$ • Efthimia Karra ${ }^{11}$. Sandip Ghosh ${ }^{7}$ - Wasim Hanif ${ }^{7}$. \\ Amy E. Edwards ${ }^{12} \cdot$ Mansoor Zafar $^{13} \cdot$ Umesh Dashora $^{13} \cdot$ Kinga A. Várnai $^{2,14}$ - Jim Davies ${ }^{2,15} \cdot$ Sarah H. Wild $^{16}$. \\ Emma G. Wilmot ${ }^{17,18} \cdot$ David Webb $^{19} \cdot$ Kamlesh Khunti $^{19} \cdot$ Rustam Rea $^{1,2}$ (D) $\cdot$ on behalf of the ABCD Covid-19 audit \\ group
}

Received: 5 February 2021 / Accepted: 23 March 2021 / Published online: 8 May 2021

(C) The Author(s) 2021

\begin{abstract}
Aims/hypothesis The aim of this work was to describe the clinical characteristics of adults with type 1 diabetes admitted to hospital and the risk factors associated with severe coronavirus disease-2019 (COVID-19) in the UK.

Methods A retrospective cohort study was performed using data collected through a nationwide audit of people admitted to hospital with diabetes and COVID-19, conducted by the Association of British Clinical Diabetologists from March to October 2020. Prespecified demographic, clinical, medication and laboratory data were collected from the electronic and paper medical record systems of the participating hospitals by local clinicians. The primary outcome of the study, severe COVID-19, was defined as death in hospital and/or admission to the adult intensive care unit (AICU). Logistic regression models were used to generate age-adjusted ORs.

Results Forty UK centres submitted data. The final dataset included 196 adults who were admitted to hospital and had both type 1 diabetes and COVID-19 on admission (male sex 55\%, white 70\%, with mean [SD] age 62 [19] years, BMI $28.3[7.3] \mathrm{kg} / \mathrm{m}^{2}$ and last recorded $\left.\mathrm{HbA}_{1 \mathrm{c}} 76[31] \mathrm{mmol} / \mathrm{mol}[9.1(5.0) \%]\right)$. The prevalence of pre-existing microvascular disease and macrovascular disease was $56 \%$ and $39 \%$, respectively. The prevalence of diabetic ketoacidosis on admission was $29 \%$. A total of 68 patients
\end{abstract}

A full list of the Association of British Clinical Diabetologists (ABCD) Study Group Collaborators can be found in the electronic supplementary material (ESM).

Rustam Rea

Rustam.rea@nhs.net

1 Oxford Centre for Diabetes, Endocrinology and Metabolism, Oxford University Hospitals NHS Foundation Trust, Oxford, UK

2 Oxford NIHR Biomedical Research Centre, Oxford, UK

3 Sandwell and West Birmingham Hospitals NHS Trust, Birmingham, UK

4 Department of Clinical \& Experimental Medicine, Faculty of Health \& Medical Sciences, University of Surrey, Guildford, UK

5 Department of Diabetes \& Endocrinology, Surrey \& Sussex Healthcare NHS Trust, Redhill, Surrey, UK

6 Medical and Dental Sciences, University of Birmingham, Birmingham, UK

7 Diabetes Centre, The Queen Elizabeth Hospital, University Hospitals Birmingham NHS Foundation Trust, Birmingham, UK

8 Department of Diabetes \& Endocrinology, Sheffield Teaching Hospitals NHS Foundation Trust, Sheffield, UK
9 Diabetes and Endocrinology Department, King's College Hospital, London, UK

10 Mid Yorkshire Hospitals NHS Trust, Pinderfields Hospital, Wakefield, UK

11 Royal Free Hospital, London, UK

12 Department of Diabetes and Endocrinology, Newham University Hospital, Barts Health NHS Trust, London, UK

13 Conquest Hospital, Hastings, UK

14 Oxford University Hospitals NHS Foundation Trust, Oxford, UK

15 Department of Computer Science, University of Oxford, Oxford, UK

16 Usher Institute, University of Edinburgh, Edinburgh, UK

17 Diabetes Department, University Hospitals of Derby and Burton NHS FT, Derby, UK

18 University of Nottingham, Nottingham, UK

19 Diabetes Research Centre, University Hospitals of Leicester NHS Trust, Leicester General Hospital, Leicester, UK 


\section{Research in context}

\section{What is already known about this subject?}

- People with diabetes and coronavirus disease-2019 (COVID-19) infection have higher risk of mortality and admission to an intensive care unit than people without diabetes

\section{What is the key question?}

- What are the clinical characteristics of adults with type 1 diabetes admitted to hospital with COVID-19 infection and the risk factors associated with risk of mortality and adult intensive care unit admission?

\section{What are the new findings?}

- In people with type 1 diabetes and COVID-19 who were admitted to hospital in the UK, higher BMI, poorer renal function and presence of microvascular complications were associated with greater risk of death and/or admission to an adult intensive care unit

- Risk of severe COVID-19 is reassuringly very low in people with type 1 diabetes who are under 55 years of age and without microvascular or macrovascular disease

\section{How might this impact on clinical practice in the foreseeable future?}

- The results from the study help to identify high-risk inpatients with type 1 diabetes and COVID-19 and gives reassurance to those who are young and without diabetes complications

(35\%) died or were admitted to AICU. The proportions of people that died were 7\%, 38\% and 38\% of those aged $<55,55-74$ and $\geq 75$ years, respectively. BMI, serum creatinine levels and having one or more microvascular complications were positively associated with the primary outcome after adjusting for age.

Conclusions/interpretation In people with type 1 diabetes and COVID-19 who were admitted to hospital in the UK, higher BMI, poorer renal function and presence of microvascular complications were associated with greater risk of death and/or admission to AICU. Risk of severe COVID-19 is reassuringly very low in people with type 1 diabetes who are under 55 years of age without microvascular or macrovascular disease.

Keywords COVID-19 $\cdot$ Inpatients $\cdot$ Mortality $\cdot$ National audit $\cdot$ Type 1 diabetes

$\begin{array}{ll}\begin{array}{l}\text { Abbreviations } \\ \text { ABCD }\end{array} & \begin{array}{l}\text { Association of British Clinical } \\ \text { Diabetologists } \\ \text { Adult intensive care unit }\end{array} \\ \text { AICU } & \begin{array}{l}\text { Coronavirus disease-2019 } \\ \text { COVID-19 }\end{array} \\ \text { DKA } & \text { Diabetic ketoacidosis } \\ \text { NDA } & \text { National Diabetes Audit } \\ \text { NHS } & \text { National Health Service } \\ \text { NIHR } & \text { National Institute for Health Research } \\ \text { OUH } & \text { Oxford University Hospitals } \\ & \text { NHS Foundation Trust } \\ \text { SARS-CoV-2 } & \text { Severe acute respiratory } \\ & \text { syndrome coronavairus-2 }\end{array}$

\section{Introduction}

The severe acute respiratory syndrome coronavirus-2 (SARSCoV-2) was first reported in Wuhan, China in December 2019 and has since been responsible for over 1.6 million deaths from coronavirus disease-2019 (COVID-19) infection globally by December 2020 [1]. A number of studies and systematic reviews have reported that certain chronic comorbidities, such as hypertension, diabetes, CVD, chronic kidney disease and chronic obstructive pulmonary disease, are associated with increased risk of severe COVID-19 and mortality [2]. Within the population of 61 million people in England, approximately $0.4 \%$ have type 1 diabetes and $4.7 \%$ have type 2 diabetes. An English population-based study reported that, up to 11 May 2020, 23,698 in-hospital COVID-19-related deaths had occurred, of which $1.5 \%$ were people with type 1 diabetes and $31.4 \%$ were people with type 2 diabetes [3]. The study estimated that ORs for in-hospital COVID-19-related death (adjusted for age, sex, deprivation, ethnicity and geographical region) was 3.51 (95\% CI 3.16, 3.90) for type 1 diabetes and $2.03(1.97,2.09)$ for type 2 diabetes compared with people without diabetes [3].

Several studies have also reported the association between admission hyperglycaemia and in-hospital mortality rate in people with diabetes. An Italian study of 59 hospitalised 
patients with COVID-19 showed that those with hyperglycaemia or diabetes had a higher mortality rate compared with those without diabetes and euglycaemia [4]. Newly diagnosed diabetes was associated with an increased mortality rate in a study of 453 patients admitted to hospital with COVID-19 in Wuhan, China (HR compared with people with normal glucose levels 9.42 [95\% CI 2.18, 40.7]) [5]. A further study of 605 COVID-19 patients admitted to two hospitals in Wuhan showed that fasting plasma glucose $>7.0 \mathrm{mmol} / \mathrm{l}$ was independently associated with higher 28 day mortality rate after adjustment for age, sex and a measure of COVID-19 disease severity (HR 2.30 [95\% CI $1.49,3.55]$ ] [6]. All these studies suggest that hyperglycaemia, including newly diagnosed diabetes, in people with COVID19 is associated with higher rates of complications and mortality. However, most studies have been small, from single centres or single cities, and have not examined the complications and mortality outcomes in a specific subset of people with diabetes. Few studies have had sufficient power to describe factors associated with poor outcomes among people with type 1 diabetes [7-9]. It is important to gain further insights into the characteristics of people with type 1 diabetes and severe COVID-19 in order to inform clinical practice and provide accurate information to people with type 1 diabetes. The aims of this study are to describe the characteristics of people with type 1 diabetes admitted to hospital with COVID19 and to identify the risk factors associated with severe disease.

\section{Methods}

Data collection Data for this retrospective cohort study were collected through a nationwide audit conducted by the Association of British Clinical Diabetologists (ABCD) [10]. A full list of ABCD Collaborators can be found in the electronic supplementary material (ESM). The nationwide audit commenced in September 2020. Contributors were asked to collate data from patient records and to transfer the data in anonymous form to the National Institute for Health Research (NIHR) Health Informatics Collaborative (HIC) Coordinating Centre within the Oxford University Hospitals NHS Foundation Trust (OUH). The data were transferred securely using the National Health Service (NHS) network. Submissions were checked by the NIHR HIC team and additional information was sought from contributing centres where necessary to ensure completeness and accuracy. The data were processed and analysed on a secure server within OUH.

Ethical approval The audit was registered with the OUH and a Data Protection Impact Assessment was carried out and approved by the OUH Caldicott Guardian and the Public
Benefit and Privacy Panel in Scotland (reference 20210111). The NHS supports audit with clear guidance for the contributing centres on the use of routine clinical practice data submitted in anonymised form via the secure NHS network. As the study was retrospective, and comprised routinely collected healthcare data only, there was no requirement for approval by a research ethics committee.

Study variables Inpatients' demographic information, clinical characteristics, medication history and laboratory measurements were collected from the electronic and paper medical record systems of the participating centres by local clinicians using a standard template. Demographic data comprised age in years, sex, ethnicity and census-derived Index of Multiple Deprivation decile. The following clinical characteristics were included: weight and height, or BMI; classification of diabetes; duration of diabetes; diabetes complications including diabetic ketoacidosis (DKA), diabetic foot ulcer, diabetic nephropathy, diabetic peripheral neuropathy, diabetic retinopathy, peripheral vascular disease, ischaemic heart disease (myocardial infarction) and/or heart failure, and cerebrovascular disease (stroke/transient ischaemic attack); and other significant comorbidities (hypertension, dementia, asthma, chronic obstructive pulmonary disease, malignant neoplasm, smoking status). Medication history included glucoselowering medications and other selected drug classes (angiotensin-converting enzyme inhibitors or angiotensin receptor antagonists, oral corticosteroids, statins, antiplatelet agents, anticoagulant agents and regular nonsteroidal antiinflammatory drugs). Laboratory data included latest preadmission $\mathrm{HbA}_{1 \mathrm{c}}$ and serum creatinine, and admission assays of blood glucose, $\mathrm{pH}$, bicarbonate, lactate, serum creatinine and capillary blood ketones. Dates of the start and finish (if applicable) of each hospital admission were collected, along with the date of positive SARS-CoV-2 test (a positive result was a prerequisite for inclusion in the study). Recorded outcomes included vital status and admission to an adult intensive care unit (AICU) during the entire hospital admission. The variables relevant to the analysis of the type 1 diabetes population were used and further data processing and analysis is ongoing for the type 2 diabetes population. The $\mathrm{ABCD}$ audit remains open and the original data collection sheet can be obtained from the $\mathrm{ABCD}$ secretariat.

Statistical analysis This report is based on people with type 1 diabetes only. Baseline clinical characteristics are reported as frequency and percentages for categorical variables, and as mean and SD for continuous variables. The primary outcome of the study was severe disease, defined as death or admission to the AICU. Mortality rate alone was a secondary outcome. Logistic regression models, with adjustment for age as a continuous variable, were used to generate ORs for the primary outcome. For the logistic regression analysis, continuous 
variables, including BMI, admission blood glucose, latest $\mathrm{HbA}_{1 \mathrm{c}}$ and serum creatinine, were transformed into categorical variables (BMI $<25$ [reference category], 25-29.9 and $\geq 30 \mathrm{~kg} / \mathrm{m}^{2}$; admission blood glucose $<10$ [reference category], $10-19.9$ and $\geq 20 \mathrm{mmol} / \mathrm{l} ; \mathrm{HbA}_{1 \mathrm{c}}<75$ [reference category] and $\geq 75 \mathrm{mmol} / \mathrm{mol}$; serum creatinine $<74,74-107$ [reference category, regarded as the normal range] and $>107 \mu \mathrm{mol} /$ 1). Binary categorical variables were also created to define sex ( 1 for male vs 0 for female), ethnicity ( 1 for non-white vs 0 for white), DKA (1 for yes), hypertension (1 for yes), dementia (1 for yes), asthma (1 for yes), chronic obstructive pulmonary disease (1 for yes), malignant neoplasm (1 for yes), smoking status ( 1 for current/previous smoker, 0 for never smoker), metformin use ( 1 for yes, 0 for no metformin), microvascular disease ( 1 for having one or more conditions from diabetic foot ulcer, diabetic nephropathy, diabetic peripheral neuropathy and diabetic retinopathy) and macrovascular disease ( 1 for having one or more conditions from peripheral vascular disease, ischaemic heart disease and cerebrovascular disease). All statistical analysis was performed using $\mathrm{R}$ version 3.3 (www.r-project.org/). A $p$ value of $<0.05$ was considered statistically significant.

\section{Results}

Demographic and clinical characteristics Up to 8 December 2020, a total of 40 NHS centres around the UK had submitted data to the ABCD COVID-19 diabetes national audit. The final dataset consisted of a total of 3312 patient records; 196 were for inpatients with type 1 diabetes who were hospitalised between March and October 2020, of whom 194 patients had primary outcome data submitted (discharged or died or admitted to ICU). All patients were classified by the local clinical teams as having type 1 diabetes and were confirmed to be receiving insulin therapy.

Table 1 summarises the clinical characteristics of the whole study population stratified by the primary outcome. There was a slightly higher proportion of men (55\%), mean (SD) age was 62 (19) years and BMI was $28.3(7.3) \mathrm{kg} / \mathrm{m}^{2}$, and the majority of people were of white ethnicity (70\%). The mean (SD) latest available $\mathrm{HbA}_{1 \mathrm{c}}$ was $76(31) \mathrm{mmol} / \mathrm{mol}(9.1$ [5.0]\%). In 80 patients (50\%), the latest available $\mathrm{HbA}_{1 \mathrm{c}}$ was $<58 \mathrm{mmol} / \mathrm{mol}$ $(<7.5 \%)$. The prevalence of diabetic retinopathy was $45 \%$ and of hypertension was $59 \%$. Overall, $56 \%$ of people had a microvascular complication (reported diabetic nephropathy, foot ulcer, retinopathy, peripheral neuropathy) and 39\% a macrovascular complication (reported peripheral vascular disease, ischaemic heart disease, cerebrovascular disease).

Table 2 describes the clinical outcomes in the whole type 1 diabetes population and in three different age groups. Twentyseven per cent of the overall population died from COVID-19 (53/194) and there was a marked age gradient, with 7\%, 38\% and $38 \%$ of people aged $<55,55-74$ and $\geq 75$ years dying, respectively. ESM Fig. 1 plots the distribution of admission durations for those patients who died in hospital. The median (IQR) time between admission and hospital death was 7 [4, 15] days. Most deaths (30 out of 53) occurred within 1 week after admission but two deaths occurred after 7 weeks. A total of 68 patients (35\%) died and/or were admitted to the AICU.

Logistic regression analysis Figure 1 shows a forest plot with ORs and 95\% CIs of death and/or AICU admission for different clinical variables after adjusting for age. ESM Table 1 reports the ORs for the primary outcome and ESM Table 2 reports the ORs for mortality rate alone. BMI, serum creatinine and presence of microvascular disease were positively associated with the primary outcome after adjusting for age while, surprisingly, higher admission blood glucose levels were negatively associated with the primary outcome after age adjustment. However, there was a statistically significant difference in the mean blood glucose value between people with DKA and those without DKA which is likely to account for this unexpected finding. In people with DKA, the mean (SD) admission blood glucose level was 29 (14) $\mathrm{mmol} / \mathrm{l}$, while in people without DKA it was $15 \pm 7 \mathrm{mmol} / \mathrm{l}(p<0.05)$.

Serum creatinine and presence of macrovascular disease were positively associated with mortality rate alone after adjusting for age (ESM Table 2).

ESM Fig. 2 shows the distribution of eGFR, with the majority of patients having abnormal pre-admission renal function (eGFR $<90 \mathrm{ml} \mathrm{min}^{-1}[1.73 \mathrm{~m}]^{-2}$ ).

\section{Discussion}

To the best of our knowledge, this study provides an analysis of the largest cohort yet assembled of people with type 1 diabetes admitted to hospital with COVID-19 infection for whom detailed clinical information is available. We report distributions of age, sex, ethnicity, diabetic complications, comorbidities, treatment on admission and laboratory test results, stratified by outcome.

The results from the present study gives reassurance to the younger type 1 diabetes population without diabetes complications with regards to their risk of severe COVID-19. However, five out of 67 (7\%) patients aged $<55$ years old died. Of these five people, three were admitted to hospital primarily for COVID-19 and two for DKA and all of them had one or both of microvascular and macrovascular complications. There were no deaths in people with type 1 diabetes under the age of 55 years old without diabetic complications.

Overall, 49 patients were admitted to hospital with DKA ( $29 \%$ of 171 patients for whom data were available). This compares with $7.3 \%$ of patients who were admitted to hospital specifically for the management of diabetes in the 2019 UK 
Table 1 Clinical characteristics of the type 1 diabetes population in the ABCD COVID-19 audit

\begin{tabular}{|c|c|c|c|c|}
\hline \multirow[t]{2}{*}{ Clinical features } & \multirow[t]{2}{*}{ Available data $(N=196)$} & \multirow[t]{2}{*}{ All } & \multicolumn{2}{|c|}{ Death and/or AICU } \\
\hline & & & Yes & No \\
\hline Male sex & 195 & 108/195 (55) & $38 / 67(57)$ & $70 / 128(55)$ \\
\hline Age, years & 194 & $62 \pm 19$ & $65 \pm 16$ & $56 \pm 21$ \\
\hline Ethnicity & 156 & & & \\
\hline White & & 109/156 (70) & $42 / 62(68)$ & 65/94 (69) \\
\hline Asian & & $12 / 156(8)$ & $4 / 62(6)$ & 8/94 (9) \\
\hline Black & & 20/156 (13) & $12 / 62(19)$ & 8/94 (9) \\
\hline Other & & $15 / 156(10)$ & $4 / 62(6)$ & 13/94 (14) \\
\hline BMI, $\mathrm{kg} / \mathrm{m}^{2}$ & 121 & $28.3 \pm 7.3$ & $28.9 \pm 7.7$ & $27.8 \pm 7.0$ \\
\hline Admission blood glucose, mmol/1 & 122 & $18.6 \pm 11.3$ & $17.3 \pm 13.5$ & $19.6 \pm 10.2$ \\
\hline $\mathrm{HbA}_{1 \mathrm{c}}, \mathrm{mmol} / \mathrm{mol}$ & 161 & $76 \pm 31$ & $66 \pm 26$ & $80 \pm 33$ \\
\hline $\mathrm{HbA}_{1 \mathrm{c}}, \%$ & 161 & $9.1 \pm 5.0$ & $8.2 \pm 4.5$ & $9.5 \pm 5.2$ \\
\hline Creatinine, $\mu \mathrm{mol} / 1$ & 137 & $142 \pm 81$ & $181 \pm 93$ & $116 \pm 71$ \\
\hline \multicolumn{5}{|l|}{ Diabetes complications } \\
\hline DKA on admission & 171 & 49/171 (29) & $14 / 64(22)$ & $35 / 107(33)$ \\
\hline Diabetic foot ulcer & 149 & $32 / 149(21)$ & $12 / 54(22)$ & 20/95 (21) \\
\hline Diabetic nephropathy & 128 & $43 / 128(34)$ & $20 / 50(40)$ & 23/78 (29) \\
\hline Diabetic peripheral neuropathy & 140 & $48 / 140(34)$ & $21 / 55(38)$ & $27 / 85(32)$ \\
\hline Diabetic retinopathy & 139 & $63 / 139(45)$ & $27 / 54(50)$ & $36 / 85(42)$ \\
\hline Microvascular disease $^{\mathrm{a}}$ & 176 & $98 / 176(56)$ & $45 / 65(69)$ & $53 / 111(48)$ \\
\hline Macrovascular disease $^{\mathrm{b}}$ & 156 & 61/156 (39) & $27 / 46(59)$ & $34 / 110(31)$ \\
\hline \multicolumn{5}{|l|}{ Comorbidities } \\
\hline Hypertension & 167 & 98/167 (59) & $40 / 65(62)$ & $58 / 102(57)$ \\
\hline Dementia & 160 & $22 / 160(14)$ & $13 / 55(24)$ & 9/105 (9) \\
\hline Asthma & 150 & 21/150 (14) & $5 / 61(8)$ & $16 / 89(18)$ \\
\hline COPD & 149 & 17/149 (11) & $7 / 61(11)$ & $10 / 88(11)$ \\
\hline Malignant neoplasm & 154 & $15 / 154(10)$ & $5 / 61(8)$ & 10/93 (11) \\
\hline \multicolumn{5}{|l|}{ Treatment on admission } \\
\hline Metformin & 161 & 19/161 (12) & $4 / 62(6)$ & $15 / 99(15)$ \\
\hline Insulin pump & 106 & 9/106 (8) & $1 / 45(2)$ & 8/61 (13) \\
\hline Smoker & 100 & 9/100 (9) & $1 / 37(3)$ & 8/63 (13) \\
\hline
\end{tabular}

Data are shown as mean $\pm \mathrm{SD}$ or $n /$ total $n(\%)$, unless stated otherwise

${ }^{a}$ Microvascular disease includes diabetic nephropathy, foot ulcer, retinopathy and peripheral neuropathy

${ }^{\mathrm{b}}$ Macrovascular disease includes peripheral vascular disease, ischaemic heart disease and cerebrovascular disease COPD, chronic obstructive pulmonary disease
National Diabetes Inpatient Audit [11]. This fourfold increase in DKA requiring hospital admission in people with type 1 diabetes is a significant concern both for the population as well as the healthcare system. Our findings emphasise the importance of supporting people with type 1 diabetes in the community during the pandemic, to minimise the incidence of avoidable admission. Health policy development may require population-specific attention to ethnicity as a risk factor for DKA, because DKA incidence varied fourfold between ethnic groups in a recently published US study [12]. We performed a
Table 2 Outcomes in patients with type 1 diabetes and COVID19 in different age groups

\begin{tabular}{llllll}
\hline Outcome & $\begin{array}{l}\text { Available } \\
\text { data }(N=196)\end{array}$ & All age groups & $<55$ years & $55-74$ years & $\geq 75$ years \\
\hline Death, $n /$ total $n(\%)$ & 194 & $53 / 194(27)$ & $5 / 67(7)$ & $29 / 77(38)$ & $19 / 50(38)$ \\
Death and/or & 194 & $68 / 194(35)$ & $17 / 72(24)$ & $32 / 71(45)$ & $19 / 51(37)$ \\
AICU, $n /$ total $n(\%)$ & & & & & \\
\hline
\end{tabular}




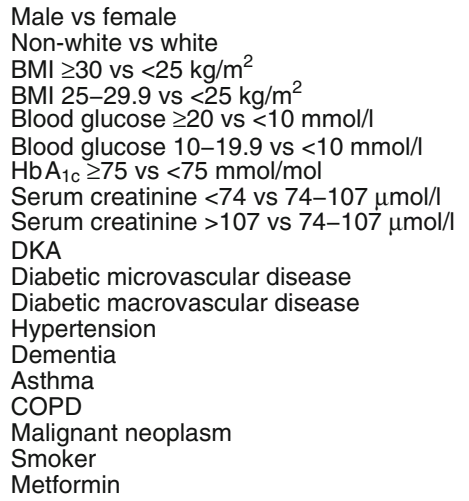

$\geq 30$ vs $<25 \mathrm{~kg} / \mathrm{m}^{2}$

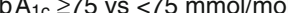

Serum creatinine $<74$ vs $74-107 \mu \mathrm{mol} / \mathrm{l}$

DKA

Diabetic macrovascular disease

Hypertension

Colignant neoplasm

Metformin

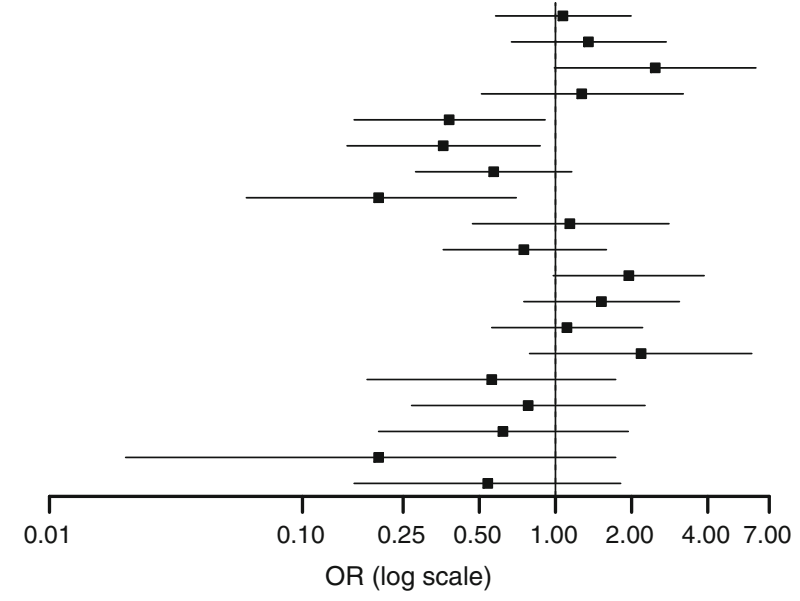

Fig. 1 Forest plot of the ORs (95\% CIs) for the association between severe COVID-19 (death or AICU admission) and clinical variables after adjusting for age. The comparison groups (sample size) are: male vs female $(n=195)$; non-white vs white $(n=156)$; BMI $\geq 30 \mathrm{~kg} / \mathrm{m}^{2}$ vs $<25 \mathrm{~kg} / \mathrm{m}^{2}(n=121)$; BMI $25-29.9 \mathrm{~kg} / \mathrm{m}^{2}$ vs $<25 \mathrm{~kg} / \mathrm{m}^{2}(n=121)$; blood glucose $\geq 20 \mathrm{mmol} / 1$ vs $<10 \mathrm{mmol} / 1 \quad(n=122)$; blood glucose 10 $19.9 \mathrm{mmol} / 1 \mathrm{vs}<10 \mathrm{mmol} / \mathrm{l}(n=122) ; \mathrm{HbA} 1 \mathrm{c} \geq 75 \mathrm{mmol} / \mathrm{mol}(\geq 9.0 \%)$ $\mathrm{vs}<75 \mathrm{mmol} / \mathrm{mol}(<9.0 \%)(n=161)$; serum creatinine $<74 \mu \mathrm{mol} / 1 \mathrm{vs} 74$ $107 \mu \mathrm{mol} / 1(n=137)$; serum creatinine $>107 \mu \mathrm{mol} / 1$ vs $74-107 \mu \mathrm{mol} / 1$

further analysis to re-examine the association between blood glucose levels and the primary outcome after adjusting for both age and DKA. The results show a non-significant association with the primary outcome for blood glucose $\geq 20 \mathrm{mmol} / 1$ vs $<10 \mathrm{mmol} / 1$ (OR 0.37 [95\% CI 0.14, 1.03], $p=0.06$ ) but the association did reach statistical significance when comparing blood glucose $10-19.9 \mathrm{mmol} / 1$ vs $<10 \mathrm{mmol} / 1$ (OR 0.35 [95\% CI 0.14, 0.89], $p=0.03$ ). We excluded four patients who were admitted to hospital with blood glucose $<4 \mathrm{mmol} / \mathrm{l}$ in this analysis. This unexpected finding of reduction in primary outcome (rate of death and AICU admission) in people with modestly elevated admission glucose compared with those with normal admission glucose needs further confirmation from a larger dataset.

We compared the clinical characteristics of this inpatient cohort with those of the general type 1 diabetes population included in the retrospective registry-based population study recently reported by the National Diabetes Audit (NDA) for England \& Wales [13]. We found similar sex and ethnicity distribution (male, $55 \%$ vs $56 \%$; white ethnicity, $70 \%$ vs $80 \%$ ) but our cohort was of older age and relatively poor glycaemic control (proportion of age $>64$ years, $45 \%$ vs $15 \%$; $\mathrm{HbA}_{1 \mathrm{c}} \leq$ $75 \mathrm{mmol} / \mathrm{mol}$ [9.0\%], $40 \%$ vs $71 \%$ ) in COVID-19 inpatients compared with the general type 1 diabetes population. The crude (i.e. not adjusted for age) prevalence of diabetesrelated complications was higher in COVID-19 inpatients than in the general type 1 diabetes population as reported in the 2017-2018 data report [14]: diabetic foot ulcer, $21 \%$ vs $5 \%$; ischaemic heart disease, $24 \%$ vs $1.9 \%$; cerebrovascular disease, $15 \%$ vs $0.5 \%$. The complications data in the NDA report are extracted from primary care data which may be up $(n=137)$; DKA vs no DKA $(n=171)$; diabetic microvascular disease vs no diabetic microvascular disease $(n=176)$; diabetic macrovascular disease vs no diabetic macrovascular disease $(n=156)$; hypertension vs no hypertension $(n=167)$; dementia vs no dementia $(n=160)$; asthma vs no asthma $(n=150)$, chronic obstructive pulmonary disease (COPD) vs no COPD ( $n=149)$; malignant neoplasm vs no malignant neoplasm $(n=$ $154)$; smoker vs non-smoker $(n=100)$; metformin vs no metformin $(n=$ 161). The base used for logarithmic transformations on the $x$-axis is $\log _{10}$

to 15 months old, whereas our findings are based on contemporaneous admission records.

To our knowledge, few studies have been published on characteristics or outcomes in people with type 1 diabetes and COVID-19. The largest study to date has been the population-based study in England (population 61 million people) describing in-hospital mortality rate among 364 people with type 1 diabetes (1.45\% of all hospital admissions due to COVID-19) [3]. The study showed that mortality was mainly confined to an older population, with no deaths occurring in those aged $<50$ years [3]. Another UK population study linked data between the NDA and the Office of National Statistics and compared the mortality rate between the year 2020 and the three previous years [15]. The study found that weekly death registrations in the first 19 weeks of 2020 exceeded the corresponding 3 year weekly averages for $2017-2019$ by $50.9 \%$ in people with type 1 diabetes and that the increased COVID-19-related mortality rate was associated with cardiovascular/renal complications of diabetes and with glycaemic control and BMI [15]. A recent Scottish population study [16] included $51(0.1 \%)$ of 34,383 people with type 1 diabetes who developed fatal or critical care unit-treated COVID-19 between 1 March 2020 and 31 July 2020. Overall, the risk of fatal or critical care unit-treated COVID19 was increased by 2.4 times in those with type 1 diabetes compared with those without diabetes. Previous hospital admissions with hypoglycaemia or DKA were strongly associated with fatal or critical care unit-treated COVD-19. Compared with the general population of people with type 1 diabetes, those in the fatal/AICU admission group were older (mean age 71.4 vs 44.5 years), had higher mean BMI (27 vs 
$26 \mathrm{~kg} / \mathrm{m}^{2}$ ), higher mean $\mathrm{HbA}_{1 \mathrm{c}}$ (69 vs $67 \mathrm{mmol} / \mathrm{mol}$ ), lower mean eGFR (72 vs $100 \mathrm{ml} \mathrm{min}^{-1}[1.73 \mathrm{~m}]^{-2}$ ), higher prevalence of heart disease (61\% vs 14\%) and lower insulin pump use ( $2 \%$ vs $14 \%)$. The patients included in this current study have not been excluded from other UK-based COVID registry studies. The present study included hospital admissions with type 1 diabetes and COVID from 1 March until 31 October 2020.

The Coronavirus-SARS-CoV-2 and Diabetes Outcomes (CORONADO) observational study from 68 French hospitals reported data for 56 people with type 1 diabetes [8]. In this cohort $55.4 \%$ were men, mean age was 56.0 years and mean BMI of $25.8 \mathrm{~kg} / \mathrm{m}^{2}$. At 7 days, 11 patients $(19.6 \%)$ had required tracheal intubation for mechanical ventilation, three had died (5.4\%) and nine (16.1\%) had been discharged. Overall, those with severe disease or who died were older (65.3 vs 53.2 years) and more likely to have hypertension (OR 5.21 [95\% CI 1.24, 21.9]) than people who had not developed these outcomes within 7 days. Poor outcomes from COVID-19 in people with type 1 diabetes in this cohort were most strongly related to age, with no deaths occurring in those aged $<65$ years [8], whereas in a larger cohort with follow-up until discharge and a different healthcare system, we found a $7 \%$ mortality rate in people aged $<55$ years. The T1D exchange quality improvement collaborative (TIDX-QI), involving 64 sites in the USA, reported data on 33 patients with type 1 diabetes and laboratory-confirmed COVID-19 [7]. Among this study population, $54.5 \%$ were female and their mean age was 24.8 years and mean $\mathrm{HbA}_{1 \mathrm{c}}$ was $69 \mathrm{mmol} / \mathrm{mol}$ (8.5\%) [7]. The most prevalent comorbidities reported in this study were obesity (39.4\%) and hypertension or CVD $(12.1 \%)$. DKA occurred in $45.5 \%$ of patients [7].

Another US study conducted a retrospective chart review in 35 people with type 1 diabetes admitted to the Beth Israel Deaconess Medical Centre in Boston, MA, of which seven had COVID-19 [9]. Although the cohort was small, the study indicated that, compared with those who were COVID-19 negative, a higher proportion of SARS-CoV-2-positive patients were of non-Hispanic Black American ethnicity, with no significant differences in sex, body weight, glucose or $\mathrm{HbA}_{1 \mathrm{c}}$ on admission. Only one patient in the COVID-19positive group and two people in the COVID-19-negative group had DKA. The study showed similar glycaemic control prior to admission among people with type 1 diabetes, regardless of COVID-19 test results [9].

Our study has several strengths. The data were collected from a large number of centres across the UK, using a structured proforma with variables prespecified based on previous studies. This is the largest published cohort of people with type 1 diabetes for whom contemporaneous admission data are available. The data include ethnicity and outcomes to discharge or death, up to 70 days from admission, reflecting the prolonged hospital course of many patients with severe
COVID-19. Limitations to our study include the retrospective nature of data collection and the absence of data from contemporaneous hospital admissions of people with type 1 diabetes without COVID-19 infection, and from people with type 1 diabetes and COVID-19 who were not admitted to hospital. We recognise that the patient population admitted to hospital are significantly different from the wider type 1 diabetes population and so the conclusions should be restricted to this subgroup of people. A number of centres had missing data for some of the variables (we report the completeness of variables in Table 1).

In conclusion, we report the largest study of people with type 1 diabetes admitted to hospital with COVID-19. In this population, higher BMI, worse renal function and the presence of microvascular complications were associated with higher risk of death and/or admission to AICU. However, no people with type 1 diabetes $<55$ years of age without microvascular or macrovascular complications died or were admitted to AICU.

Supplementary Information The online version contains peer-reviewed but unedited supplementary material available at https://doi.org/10.1007/ s00125-021-05463-x.

Acknowledgements We are grateful to all the people who collected the data for this study, to B. Maylor and J. Miksza (both Leicester Diabetes Centre, Leicester, UK) for data template development, and to M. Cull of the $\mathrm{ABCD}$ secretariat for administrative support.

Data availability The ABCD audit remains open and the original data collection sheet can be obtained from the ABCD secretariat. Data are available on request from the authors.

Funding This research received no specific grant from any funding agency in the public, commercial or not-for-profit sectors. YR acknowledges support from a Novo Nordisk Postdoctoral Fellowship run in partnership with the University of Oxford. KK acknowledges support from the NIHR Applied Research Collaboration East Midlands (ARC EM) and the NIHR Leicester Biomedical Research Centre (BRC). RR is supported by the NIHR Oxford Biomedical Research Centre (BRC).

Authors' relationships and activities RR has acted as a consultant, speaker or received grants from Novo Nordisk, Eli Lilly and Boehringer Ingelheim. KK has acted as a consultant, speaker or received grants for investigator-initiated studies for Astra Zeneca, Novartis, Novo Nordisk, Sanofi-Aventis, Lilly and Merck Sharp \& Dohme, Boehringer Ingelheim, Bayer, Berlin-Chemie AG/Menarini Group, Janssen and Napp. EGW has received personal fees from Abbott Diabetes Care, Dexcom, Diasend, Eli Lilly, Insulet, Medtronic, Novo Nordisk and Sanofi-Aventis. SHW attends meetings of the Scottish Study Group of Diabetes in the Young that receives support from Novo Nordisk. SH has received educational funding support from Sanofi-Aventis and consulting fees from Eli Lilly and Oviva. AI has consulted for OrbiMed LLC and received educational grant support from Sanofi SA. BCTF has acted as a consultant, speaker or received grants from Abbott Diabetes, AstraZeneca, Boehringer Ingelheim, Eli Lilly, GSK, Janssen, Medtronic, MSD, Napp, Novo Nordisk and Sanofi. UD has acted as a speaker for educational talks and received lecture fee from Astra Zeneca, Novartis, Novo Nordisk, Sanofi-Aventis, Lilly and Merck Sharp \& Dohme, Boehringer Ingelheim, Janssen and Napp. REJR has received speaker fees and/or consultancy fees and/or educational sponsorships 
from AstraZeneca, BioQuest, GI Dynamics, Janssen, Novo Nordisk, Sanofi-Aventis and Takeda. All the other authors listed in this paper declare that there are no relationships or activities that might bias, or be perceived to bias, their work.

Contribution statement YR and RR co-designed the study analysis. YR carried out the data analysis. BCTF developed the data collection template. YR, KK, SHW, SH, EGW and RR drafted the manuscript. All authors contributed to the conception and design of the study, interpretation of the data and critical review of the paper and gave final approval of the version to be published. RR and YR are the guarantors of this work and, as such, had full access to all the data in the study. RR and YR are responsible for the integrity of the work as a whole.

Open Access This article is licensed under a Creative Commons Attribution 4.0 International License, which permits use, sharing, adaptation, distribution and reproduction in any medium or format, as long as you give appropriate credit to the original author(s) and the source, provide a link to the Creative Commons licence, and indicate if changes were made. The images or other third party material in this article are included in the article's Creative Commons licence, unless indicated otherwise in a credit line to the material. If material is not included in the article's Creative Commons licence and your intended use is not permitted by statutory regulation or exceeds the permitted use, you will need to obtain permission directly from the copyright holder. To view a copy of this licence, visit http://creativecommons.org/licenses/by/4.0/.

\section{References}

1. Worldometer. Coronavirus. Available from: www.worldometers. info/coronavirus. Accesed 15 Dec 2020

2. Singh AK, Gillies CL, Singh R et al (2020) Prevalence of comorbidities and their association with mortality in patients with COVID-19: a systematic review and meta-analysis. Diabetes Obes Metab 22(10):1915-1924. https://doi.org/10.1111/dom. 14124

3. Barron E, Bakhai C, Kar P et al (2020) Associations of type 1 and type 2 diabetes with COVID-19-related mortality in England: a whole-population study. Lancet Diabetes Endocrinol 8(10):813822. https://doi.org/10.1016/S2213-8587(20)30272-2

4. Marfella R, Paolisso P, Sardu C et al (2020) Negative impact of hyperglycaemia on tocilizumab therapy in Covid-19 patients. Diabetes Metab 46(5):403-405. https://doi.org/10.1016/j.diabet. 2020.05.005

5. Li H, Tian S, Chen T et al (2020) Newly diagnosed diabetes is associated with a higher risk of mortality than known diabetes in hospitalized patients with COVID-19. Diabetes Obes Metab. https://doi.org/10.1111/dom.14099
6. Wang S, Ma P, Zhang S et al (2020) Fasting blood glucose at admission is an independent predictor for 28-day mortality in patients with COVID-19 without previous diagnosis of diabetes: a multi-centre retrospective study. Diabetologia. 63(10):2102-2111. https://doi.org/10.1007/s00125-020-05209-1

7. Ebekozien OA, Noor N, Gallagher MP, Alonso GT (2020) Type 1 diabetes and COVID-19: preliminary findings from a multicentre surveillance study in the U.S. Diabetes Care 43(8):e83-ee5. https:// doi.org/10.2337/dc20-1088

8. Wargny M, Gourdy P, Ludwig L et al (2020) Type 1 diabetes in people hospitalized for COVID-19: new insights from the CORONADO study. Diabetes Care 43(11):e174-e1e7. https://doi. org/10.2337/dc20-1217

9. Vamvini M, Lioutas VA, Middelbeek RJW (2020) Characteristics and diabetes control in adults with type 1 diabetes admitted with COVID-19 infection. Diabetes Care 43(10):e120-e1e2. https://doi. org $/ 10.2337 / \mathrm{dc} 20-1540$

10. ABCD. ABCD COVID-19 \& Diabetes: UK national audit Available from: www.diabetologists-abcd.org.uk/Coronavirus/ COVID-19_Diabetes_Nationwide_Audit.html. Date accessed 7 Dec 2020

11. NHS Digital. National Diabetes (2020) Inpatient Audit (NaDIA) 2019. Available from: https://digital.nhs.uk/data-and-information/ publications/statistical/national-diabetes-inpatient-audit/2019. Accessed: 20 Jan 2021

12. Osagie E, Shivani A, Nudrat $\mathrm{N}$ et al (2021) Inequities in diabetic ketoacidosis among patients with type 1 diabetes and COVID-19: data from 52 US clinical centres. J Clin Endocrinol Metab 106(4): e1755-e1762

13. NHS Digital. (2020). Care Processes and Treatment Targets, January to December 2019. Available from: https://digital.nhs.uk/ data-and-information/publications/statistical/national-diabetesaudit/national-diabetes-audit-quarterly-report-january-todecember-2019. Accessed: 30 Dec 2020

14. NHS Digital (2019). National Diabetes Audit, 2017-18: Report 2a: Complications and Mortality (complications of diabetes). England and Wales. Available from: https://files.digital.nhs.uk/91/084B1D/ NationalDiabetesAudit\%2C2017-18\%2CReport2a.pdf. 2020. Accessed 6 Jan 2021

15. Holman N, Knighton P, Kar P et al (2020) Risk factors for COVID19-related mortality in people with type 1 and type 2 diabetes in England: a population-based cohort study. Lancet Diabetes Endocrinol 8(10):823-833. https://doi.org/10.1016/S22138587(20)30271-0

16. Stuart M, Amanda W, Jen B et al (2020) Risk of and risk factors for COVID-19 disease in people with diabetes: a cohort study of the total population of Scotland. Lancet Diabetes Endocrinol 9(2):8293

Publisher's note Springer Nature remains neutral with regard to jurisdictional claims in published maps and institutional affiliations. 